\title{
Redes sociales y TIC, su papel en la educación superior del siglo XXI
}

\author{
María Yolanda MarTínez SolanA \\ Universidad Complutense de Madrid \\ mymartínez@ccinf.ucm.es
}

\begin{abstract}
Resumen:
El siglo XXI ha supuesto una transformación en el ámbito cultural de las sociedades, fruto del surgimiento de diferentes maneras de generar conocimiento y de transmitir información que ha afectado a todos los ambientes académicos. Los docentes están obligados a formarse en el uso, conceptos y alcances de las redes sociales y las TIC aunque estas no pueden reemplazar las herramientas educativas preexistentes. Todo ello implica un replanteamiento del sistema educativo que permita hablar de una verdadera tecnología educativa. La aparición del Ágora digital ha derribado fronteras de acceso a los contenidos pero construido muros de compartimentación de los conocimientos y un uso inadecuado de las nuevas tecnologías puede acarrear consecuencias indeseadas.
\end{abstract}

Palabras clave: Redes sociales; TIC; Educación superior; Ágora digital; Docentes; Conocimiento.

\section{Social Networks and ICTs, their role in 21st century higher education}

\begin{abstract}
:
21 st century has brought a transformation of the cultural context of societies, as a result of the appearance of different sources of knowledge and means of information transmission, which have affected all academic environments. Instructors are forced to be trained in use, concepts and range of social networks and ICTs, even though these may not substitute the pre-existent educational tools. This leads to a redefinition of the educational system, in order to embrace a genuine educational technology. The birth of the Digital Agora has knocked down the limits to content access while, at the same time, compartmentalizing knowledge, and the inadequate use of new technologies might entail non-desirable consequences.
\end{abstract}

Key words: Social Networks; ICT; Higher Education; Digital Agora; Instructors; Knowledge.

\section{Referencia normalizada:}

Martínez Solana, M. Y. (2014): Redes sociales y TIC, su papel en la educación superior del siglo XXI. Historia y Comunicación Social. Vol. 19. Núm. Especial Marzo. Págs. 63-71.

Sumario: 1. Introducción. 2. TIC y redes sociales en el campo educativo. 3. La educación como acto social. 4. Conclusiones. 5. Referencias bibliográficas. 


\section{Introducción}

Actualmente las Nuevas Tecnologías de la Información (TIC) hacen parte del día a día de muchas personas en el mundo, realidad que ha conllevado una transformación en el ámbito cultural de las sociedades, fruto del surgimiento de diferentes maneras de generar conocimiento y de transmitir información (Caritá, Padovan y Pereira, 2011). Por consiguiente, estas nuevas herramientas están jugando cada día un papel más protagónico en las aulas de clase y en general en los distintos ambientes académicos y de formación.

Ya que acceder a la información no implica, per se, acceder al conocimiento, de la misma forma, poseer estas herramientas en el aula, o convertirlas en la propia aula, no implica calidad en la educación. Así, si estas herramientas tecnológicas agregan nuevos elementos a la educación, es necesario investigar el fenómeno para mejorar sus competencias educativas (Suarez Guerrero, 2010). Estudiar el desarrollo de las TIC y su influencia en la educación actual es, por tanto, una tarea argumentada y necesaria para reforzar las bondades de esta unión y mitigar los yerros de la misma.

Internet ha sufrido fuertes mutaciones en cortos lapsos. La introducción de las web 2.0 han transformado la comunicación dentro de esta red en un proceso circular, superando la limitada comunicación lineal a la que en un principio estaba restringida y convirtiendo a los internautas en prosumer simultáneamente consumidores y productores de contenido. Llegamos a actuaciones de comunicación centradas en el diálogo, superando aquellas limitadas al discurso (Tuñez, Martínez Solana y Abejon, 2010).

Esta nueva simbiosis afecta tanto al escenario como a los actores del mismo, lo que implica una necesidad de que los maestros se formen en el uso, conceptos y alcances de las redes sociales y las TIC, de tal manera que las puedan aplicar de una manera adecuada, más aun cuando la aparición de estas nuevas herramientas ha hecho surgir una nueva forma de relación entre el docente y el estudiante. Sin embargo, no hay que olvidar que las TIC complementan pero no reemplazan las anteriores herramientas educativas.

Las nuevas tecnologías han derribado barreras de acceso a los contenidos pero construido muros de compartimentación de los conocimientos, no puede caerse en el error de relegar las relaciones interpersonales en el entorno educativo. Educar en el uso apropiado de las TIC y las redes sociales se presenta imperativo, hasta que no se haga un uso racional, ordenado, creativo y bien planeado de estas herramientas en los ambientes educativos no podemos decir que estamos frente a una verdadera "tecnología educativa". 


\section{TIC y redes sociales en el campo educativo}

Las diversas maneras de interactuar del docente durante el proceso educativo determinan y entretejen diversas acciones comunicativas, con el fin de generar procesos de aprendizaje. Por su parte, las nuevas herramientas de la comunicación han reorientado los ámbitos sociales, económicos, científicos, políticos (Caritá et al., 2011), y en general las relaciones interpersonales, lo que se traduce, por supuesto, en transformaciones en las perspectivas educativas, que, por consiguiente, exigen una evolución y adaptación de la práctica docente.

Para José Ignacio Aguaded (2001), las TIC se pueden explotar dentro del aula como recursos didácticos, cumpliendo un papel transversal de auxiliares pedagógicos; como medios de creación y expresión, para producir mensajes audiovisuales y multimediáticos; como contenido curricular, dentro de la educación formal; o como medios de desarrollo comunitario, vinculando la comunidad académica con la sociedad en general.

Gran cantidad de docentes universitarios consideran que el impacto de las TIC en el proceso enseñanza-aprendizaje es positivo, por los beneficios que ello implica en el proceso educativo e, incluso, algunos señalan que "estas herramientas fortalecen considerablemente la aprehensión de conocimiento y el enriquecimiento cognitivo" (Riascos, Quintero y Ávila, 2009: 145). En este sentido, una correcta vinculación de las TIC a este proceso "abre nuevas posibilidades para complementar la educación formal" (Caritá et al., 2011, traducido)'. No obstante "La integración de las TIC en modelos formativos no adecuados no solo no mejora el aprendizaje sino que lo empeora incrementando la carga del profesorado y de los estudiantes" (Rodríguez, 2011: 2).

Varias dificultades se han registrado en varios estudios sobre las nuevas tecnologías como herramienta del proceso educativo "como el acceso restringido a la tecnología y a los servicios de apoyo, la falta de habilidades tecnológicas, la falta de una comunidad, pobres habilidades de lectura y académicas y resistencias en el manejo de las tecnologías", consecuencias del "abuso del uso por encima de la reflexión pedagógica, la falta de formación y velocidad de los cambios" (Rodríguez, 2011:3).

De manera más específica, las redes sociales, entendidas como servicios web que permiten a los individuos construir un perfil público o semi-público dentro de un sistema delimitado, articular una lista de contactos y ver y cruzar su lista de conexiones y las hechas por otros dentro del sistema (Boyd y Ellison, 2007), son un medio muy útil para "compartir todo tipo de datos e información y en múltiples formatos: audio, texto y vídeo, y con muchas menos limitaciones que el correo electrónico, cuya capacidad es mucho más limitada" (Abuín, 2009). Estas nuevas herramientas brindan una amplia capacidad de comunicación que facilita el fluido de información de toda clase, ya que si bien en un principio se utilizaban para publicar, esencialmente, reflexiones o vivencias de los usuarios, hoy en día casi todos los informativos 
y grandes medios de comunicación cuentan con perfiles en esta redes sociales (Caritá et al., 2011).

No todas las redes sociales son de la misma naturaleza ni ofrecen las mismas herramientas; las hay educativas, académicas y profesionales, por poner algunos ejemplos. Pizarra, una red social creada en Bolivia y cuyo objetivo es poner en contacto la comunidad académica, permitiendo "ejercer el rol de alumno o profesor, compartiendo sus conocimientos, sus trabajos o su experiencia con los demás miembros de la comunidad", y EDU 2.0 desarrollada por Graham Glass, que "pretende dar una respuesta a las necesidades y metodologías educativas actuales, ofreciendo a los docentes y profesionales del sector una plataforma gratuita con más 10.000 recursos educativos aportados por otros docentes de todo el mundo", son ejemplos de redes académicas (Abuín, 2009).

Facebook, la red social más popular, fue creada en febrero de 2004 en un ambiente académico, la Universidad de Harvard en los EEUU por Mark Zuckerber y tres amigos suyos (Kirkpatrick, 2010). Actualmente, esta red social cuenta con casi 1.000 millones de usuarios registrados, más de 250 millones en Europa y 17 millones de usuarios registrados en España, es decir, alrededor del 37\% de la población española y el 57\% de sus usuarios de Internet, según la web Socialbakers. Asimismo, la mayoría de los usuarios registrados en España están comprendidos entre los 25 y 34 años de edad, alrededor de 5’200.000. Facebook ha ganado la preferencia de los usuarios de Internet para compartir ideas, noticias y productos, por medio de sus aplicaciones, ya que, entre otras razones, tiene como característica la centralización de informaciones, al igual que permite el acceso a través de la telefonía móvil, disminuyendo la distancia entre la red y el usuario (Caritá et al., 2011).

Los investigadores Miguel Tuñez y José Sixto han llegado a concluir, luego de estudiar la utilidad docente de las redes sociales, que la docencia encaja en los posibles usos de Facebook, aunque se trate de una red para comunicación personal y social, ya que no se considera una injerencia en ámbitos privados y en que se califica como soporte recomendable para relaciones docentes. No obstante, apuntan que no encaja con actividades obligatorias, debido a que no puede obligarse a los alumnos a que se registren en una red social. Así, sin ser plataforma docente, Facebook es un espacio académicamente útil que puede adaptarse a actividades de formación académica, donde los contenidos son pro-activos, hay una alteración de roles.

Igualmente, para Tuñez y Sixto, la red social es un punto de encuentro colectivo atemporal, por lo que no está pensada para medir la actividad bajo parámetros académicos, donde el proceso educación-aprendizaje no se limita a los días de clase presencial y los contenidos tienen una fecha de caducidad muy corta, ya que en muchos casos sólo se consume lo que se ve.

Finalmente, para los investigadores, la utilización de Facebook requiere una adaptación del método docente: diálogo breve, no discursos o lecciones magistrales, que representa trabajo extra para el docente y para el alumno, si este último así lo desea. En todo caso, y sobre todo, ni Facebook ni las demás redes sociales reemplazan 
la formación presencial u otras modalidades de formación a distancia, sino que las complementan.

\section{La educación como acto social}

Los centros de educativos son espacios de convivencia donde se combinan los campos político, social y económico, por lo que la formación profesional, en las universidades, es solamente una parte de la formación en su conjunto. Los fines de la formación superior en este siglo, según la UNESCO, son dos: "educar, formar y realizar investigaciones" y, en el aspecto ético, infundir "autonomía, responsabilidad y prospectiva" (1998), apostando no sólo por formar "diplomados altamente cualificados", sino "ciudadanos que participen activamente en la sociedad" y que posean una preparación tal que les facilite suplir las necesidades de la sociedad.

En este sentido, saber desempeñar una profesión debe suponer una educación contextualizada, que permita mejorar la calidad de vida de la población y que promueva el desarrollo humano. La educación es un acto social, por lo que no puede desligarse de los demás ámbitos que articulan a la sociedad. Es decir, el aprendizaje social abarca los contextos formales y los informales.

Dentro de los contextos informales claramente tenemos aquellos mediados por las redes sociales, que pueden ser entendidas como una forma de entidad social más fluida en la cual sus miembros de juntan, entran, salen y producen con facilidad, diferentes de un grupo que está definido como tal en un ambiente formal a partir de intenciones e intereses comunes. Las redes sociales estampan una nueva metodología de actuación y oportunidades de lazos más amplios, por lo que se presenta necesario, bajo este orden de ideas, analizar tanto el contenido como el contexto en el proceso educación-aprendizaje.

Las innovaciones tecnológicas suelen traer consigo inquietudes respecto a su idoneidad más que respecto a su correcta utilización, llevando a extensas discusiones que dejan de lado los aspectos centrales, mientras su desarrollo y aplicación continúa. Realmente, como resalta Rodríguez: "Los cambios más profundos que se nos están demandando no son solamente tecnológicos, sino más bien de mentalidad, actitudinales y de formación; de ahí la dificultad de producir cambios instantáneos en la educación" (2011: 11).

Asimismo, como señalan Tuñez y Sixto, compete al docente gestionar la estrecha línea que divide lo personal y lo profesional en el mundo de las redes sociales, ya que con estas existe "la posibilidad de usar los ámbitos de comunicación personal y social para el establecimiento de flujos de formación académica, fomentando, preferentemente, usuarios creadores y críticos" (2011).

Otro aspecto a tener en cuenta en este análisis, es el de evitar, como sucede en muchos casos, utilizar las redes sociales para expresarse de la misma manera que en 
la educación formal, llevando a los estudiantes simplemente a escribir y publicar los archivos en la red. Como señala Rodríguez: "no es difícil encontrar cursos en los que su virtualización ha consistido exclusivamente en poner a disposición a través de la web los apuntes en formato electrónico. En este esquema, el estudiante sigue siendo el mismo espectador pasivo que era antes y además ahora se encuentra solo ante un aparato y frente a las autopistas de la información lo que le puede llevar a sentirse perdido" (2011: 2). No obstante, el potencial de las TIC y de las redes sociales va mucho más allá, por lo que hay que apropiarse de la dinámica propia del aprendizaje informal y traerla para las configuraciones formales o crear un ambiente de aprendizaje informal.

Si bien las redes sociales aún no son accesibles para el cien por ciento de la población, cada día están llegando a más sectores de la sociedad y con más facilidades, ya que, por ejemplo, ahora es posible acceder a ellas de forma permanente a través de los dispositivos móviles, lo que a su vez aumenta el volumen de información en las redes. El desafío se encuentra en formar a los usuarios para que puedan filtrar el contenido de la información que reciben de una forma adecuada, ya que, como señala Aguaded, "el aprendizaje es un proceso complejo en el que existen diferentes estructuras de mediación entre las que sobresale especialmente el propio aprendiz que es el que ha de filtrar los estímulos, organizarlos, procesarlos y asimilarlos para poder integrarlos y reaccionar a otros estímulos externos con ellos" (2001).

Asimismo, es preciso que los estudiantes "«construyan» sus aprendizajes, para lo que las nuevas tecnologías, desde una adecuada orientación docente, pueden tener un papel relevante, si enseñamos a descubrir sus códigos y lenguajes y los convertimos en adecuados medios, que más que la hipnosis, fomenten el aprendizaje significativo" (Aguaded, 2001).

Se entiende, por tanto, que las nuevas herramientas comunicativas por sí solas no contribuyen al proceso educativo, ya que "cómo las utilicemos, para qué y en qué contexto es lo que hace que tengan una incidencia u otra" (Rodríguez, 2011: 2). Como señala Aguaded: "Los alumnos no aprenden por la mera seducción tecnológica de los aparatos, ni por su novedad, ni por su estela de futurismo" (2001), la utilización de estas tecnologías en el proceso educativo debe venir acompañado de una orientación que proporcione al estudiante la capacidad de hacer un adecuado uso de las mismas.

La educación superior debe educar al estudiante para que "comprenda lo que está aprendiendo y lo que está haciendo, que lo conceptualice, que pueda dominarlo bajo distintos ángulos, que pueda evaluarlo y que tome una posición crítica en relación a sus aprendizajes para determinar su relevancia para sí y su entorno" (Gutierrez, 2010: 48), lo que requiere de una redefinición de las funciones de los componentes que constituyen el sistema, de lo contrario el uso de las nuevas tecnologías en el ambiente formativo no evolucionará sino que acarreara resultados adversos (Rodríguez, 2011).

En definitiva, las nuevas herramientas de la comunicación y en especial las redes sociales, deben ser un apoyo para el proceso educativo no un sinónimo de superficialidad en la formación, más aún cuando "Nuestra sociedad precisamente se caracteriza 
por la instantaneidad icónica y por ello es que cada vez se hace más difícil argumentar rigurosos planteamientos y actuaciones" (Aguaded, 2001). Como señala Rodríguez: "La clave, como se ve, está en la promoción y estímulo hacia un tipo de aprendizaje, profundamente humano, significativo y no superficial" (2011: 20).

\section{Conclusiones}

Primera- La educación debe acomodarse a las exigencias actuales de la sociedad, por medio de un proceso analizado, planeado y realizado de tal manera que realmente sea beneficioso para el proceso educativo.

Segunda- La incidencia de las nuevas tecnologías en el proceso educativo está obligando a los actores de la educación a cambiar los procesos y las herramientas con que se construye conocimiento, por lo que los educadores deben capacitarse en conceptos, procedimientos y actitudes de la tecnología, y deben centrar su atención en formar estudiantes autónomos y críticos que manejen responsablemente los recursos tecnológicos, lo que implica un replanteamiento del sistema educativo que permita hablar de una verdadera tecnología educativa.

Tercera- Hay que volver al para qué de la educación y no limitarnos al cómo. Como acto social, el proceso educativo no sólo debe estar articulado a los demás ámbitos de la sociedad sino que debe formar ciudadanos capaces de transformarla positivamente.

Cuarta- Las TIC, en general, y las redes sociales, en particular, deben ser un apoyo para el proceso educativo no un sinónimo de superficialidad.

Quinta- Un uso adecuado de las nuevas tecnologías de la comunicación, sobre todo de las redes sociales, puede ser de gran utilidad para el proceso educativo. Sin embargo, un uso inadecuado puede acarrear consecuencias indeseadas.

Sexta- El nuevo Ágora digital ha derribado fronteras de acceso a los contenidos pero construido muros de compartimentación de los conocimientos, por ello, no se puede caer en el error de sustituir las relaciones interpersonales en el entorno educativo por las vicarias, a través de las nuevas tecnologías.

\section{Bibliografía}

ABUÍN VENCES, N. (2009). "Las redes sociales como herramienta educativa en el ámbito universitario". IV Jornada de Innovación Pedagógica del Proyecto ADA-Madrid. Desarrollo de competencias a través de la red. Madrid: Grupo de investigación FONTA, Universidad Complutense de Madrid.

AGUADED GÓMEZ, José Ignacio (2001). “Aprender y enseñar con las tecnologías de la comunicación". Ágora digital, No 1.(Ejemplar dedicado a: La Educación del futuro, el futuro de la Educación) disponible en http://www.uhu.es/agora/digital/ numeros/numeros_ppal.htm [fecha de consulta: 21-01-2013]. 
BOYD, Danah M. y ELLISON, Nicole B. (2007). "Social network sites: Definition, history, and scholarship". Journal of Computer-Mediated Communication, 13(1), article 11, en http://jcmc.indiana.edu/vol13/issue1/boyd.ellison.html [fecha de consulta: 18 de enero de 2013].

CARITÁ, Edilson Carlos; PADOVAN, Victor de Toni y PEREIRA S., Leandro Manuel. (2011). "Uso de redes sociais no processo ensinoaprendizagem: avaliação de suas características", en http://www.abed.org.br/congresso2011/cd/61.pdf [fecha de consulta: 20 de enero de 2013].

GARCIA SANS, A. (2009). "Las redes sociales como herramientas para el aprendizaje colaborativo: una experiencia con Facebook. Re-Presentaciones: Periodismo", Comunicación y Sociedad, (5), 48-63.

GUTIÉRREZ PANTOJA, Gabriel. (2010). "Los fines y opciones de la educación superior a lo largo de la historia". Revista de Antropología Experimental, $\mathrm{N}^{\circ} 10$, Especial Educación 4. Jaén: Universidad de Jaén.

KIRKPATRICK, David. (2010). "The Facebook effect: the inside story of the company that is connecting the word". New York: Simon\&Schuster. pp. 19-41.

RIASCOS ERAZO, Sandra Cristina; QUINTERO CALVACHE, Diana María y ÁVILA FAJARDO, Gloria Patricia. (2009). "Las TIC en el aula percepciones de los profesores universitarios". Educación y Educadores, volumen 12, número 3. Cali, Colombia: Universidad del Valle, Informática educativa. pp. 133-157.

RODRÍGUEZ IZQUIERDO, Rosa María. (2011). "Repensar la relación entre las TIC y la enseñanza universitaria: problemas y soluciones". Profesorado, Revista de currículum y profesorado, volumen $15, \mathrm{~N}^{\circ}$ 1, Sevilla: Universidad de Olavide (UPO), pp. 2-20.

SOCIALBAKERS. En www.socialbaker.com [fecha de consulta: 24 de enero de 2013]. SUAREZ GUERRERO, Cristóbal. (2010). "La formación en red como objeto de estudio" [artículo en línea]. Revista de Universidad y Sociedad del Conocimiento (RUSC). Vol. 7, n. ${ }^{\circ}$ 2. UOC. [Fecha de consulta: 17 de febrero de 2013].

http://rusc.uoc.edu/ojs/index.php/rusc/article/view/v7n2-suarez/v7n2-suarez

TUNNEZ, Miguel y SIXTO, José. (2011). "Redes sociales, política y Compromiso 2.0: la comunicación de los diputados españoles en Facebook". Revista Latina de Comunicación Social, n. 66. http://www.revistalatinacs.org/11/art/930_Santiago/09 Tunez.html. [Fecha de consulta: 18 de febrero de 2013].

TÚÑEZ, Miguel y SIXTO José. (2012). "Las redes sociales como entorno docente: análisis del uso de Facebook en la docencia universitaria". Píxel-Bit: Revista de Medios y Educación, 41, 77-92. http://acdc.sav.us.es/pixelbit/images/stories/p41/06. pdf [fecha de consulta: 14 de febrero de 2013].

TÚÑEZ, Miguel. (2010). "Los periódicos en las redes sociales: audiencias, contenido, interactividad y estrategias comerciales", Estudios del Mensaje Periodístico, vol. 18, p. 224. http://acdc.sav.us.es/pixelbit/images/stories/p41/06.pdf http:// revistas.ucm.es/index.php/ESMP/article/view/39367 [Fecha de consulta: 17 de febrero de 2013] 
UNESCO. (1998). “La educación superior en el siglo XXI: Visión y acción”. En http:// www.unesco.org/education/educprog/wche/declaration_spa.htm [fecha de consulta: 26 de enero de 2013].

\section{Notas}

1 Texto original: "TIC para a aprendizagem abre novas possibilidades para complementar a educação formal".

\section{La autora}

María Yolanda Martínez Solana Doctora en Periodismo y Profesora de la Facultad de CC. de la Información de la Universidad Complutense de Madrid. Experta en Información sobre Ciencias de la Salud y aplicación de la comunicación en instituciones públicas y privadas. Autora de varios libros y artículos científicos en relación con estos temas. Profesora invitada y ponente en diversos cursos y actos organizados por universidades españolas y otras instituciones. Miembro del consejo editorial de la revista "Estudios sobre el Mensaje Periodístico" de la UCM.

Ejercicio ininterrumpido de la actividad periodística en diferentes medios de comunicación y asesora de comunicación en organismos públicos y empresas. 